\section{Weiblich, jung und ADHS}

Bei Mädchen mit ADHS gibt es wenige Follow-up-Studien und keine davon hat die Patientinnen jemals bis ins Erwachsenenalter begleitet. US-amerikanische Wissenschaftler holten dies nun nach.

W issenschaftler vom Massachusetts General Hospital aus Boston nahmen insgesamt 140 Mädchen mit Aufmerksamkeitsdefizit-/Hyperaktivitätsstörung (ADHS) und 122 Mädchen ohne ADHS in ihre Untersuchung auf. Die Mädchen waren zwischen sechs und 18 Jahre alt. Elf Jahre nach der Erstuntersuchung wurden die Mädchen erneut mit Hilfe von strukturierten Interviews (KSADS-E) evaluiert. Es konnten noch 96 (69\%) Mädchen mit ADHS und 91 (75\%) ohne ADHS erfasst werden. Das mittlere Alter betrug zu diesem Zeitpunkt 22 Jahre. Die Untersuchungen ergaben, dass die jungen Mädchen mit ADHS ein signifikant höheres Risiko für das Auftreten und Persistieren von psychischen (Depressionen, Angst- und Essstörungen) und sozialen Störungen (antisoziales Verhalten sowie erhöhte Delinquenz) zeigten.
Kommentar: Durch zahlreiche Studien ist das erhöhte Risiko für junge Erwachsene mit ADHS für soziale und psychische Störungen bekannt. Mit der Studie von Biederman und Kollegen liegt erstmals eine Untersuchung von jungen Frauen mit ADHS vor. Wie auch junge Männer, haben junge Frauen eine signifikant schlechtere Prognose hinsichtlich psychosozialer Störungen als junge Erwachsene ohne ADHS. Bisher wenig beachtet werden die Essstörungen: In der Studie litten junge Frauen mit ADHS signifikant häufiger sowohl an Anorexie als auch an Bulimie mit einem Morbiditätsrisiko von $0,2 \mathrm{im}$ Vergleich zu 0,05 bei jungen Frauen ohne ADHS. Im klinischen Alltag wird auch oft extremes Übergewicht bei von ADHS betroffenen Erwachsenen beobachtet.
Leider gibt die Studie nicht darüber Auskunft, ob eine effektive Therapie der ADHS das Risiko senkt, an psychosozialen Störungen zu erkranken. Die Tatsache, dass junge Frauen mit ADHS stärker gefährdet sind, an psychischen und sozialen Störungen zu erkranken, sollte jedoch dazu motivieren, bei Auftreten dieser Störungen ein ADHS auszuschließen. Umgekehrt sollte immer gezielt bei der Diagnose ADHS nach dem gleichzeitigen Bestehen von anderen psychischen und sozialen Störungen gesucht werden. Da die Möglichkeit nicht auszuschließen ist, dass durch eine rechtzeitige und effektive Therapie der ADHS das Auftreten der psychischen und sozialen Störungen verhindert werden kann, sollte diese Diagnose bei Mädchen nicht übersehen werden.

Dr. Kirsten Stollhoff

Biederman J et al. Adult psychiatric outcomes of girls with attention deficit hyperactivity disorder: 11-year follow-up in a longitudinal case control study. Am J Psychiatry 2010; 167: 409-17

\title{
Bekommen deutsche Kinder zu oft Homöopathika?
}

In den letzten Jahren wurde in einigen europäischen Ländern und auch in den USA eine Art Renaissance der Homöopathie beobachtet: Immer mehr Patienten lassen sich homöopathisch behandeln. Deutschland ist das Mutterland dieser Therapieform. Eine Studie des Robert-Koch-Institutes hat jetzt untersucht, wie hoch hier der Anteil homöopathischer Behandlungen bei Kindern ist.

E ine große Umfrage (KiGGS) an ¿ 17.450 deutschen Kindern (Alter 0-17 Jahre) brachte interessante Daten zur Einnahme von Homöpathika in dieser Altersgruppe. Gefragt wurde, ob und welche Arzneimittel das Kind innerhalb der letzten sieben Tage eingenommen hatte.

4,6\% der Zielpopulation hatten Homöopathika eingenommen. In $11 \%$ der Fälle wurden sie als Schnupfenmittel gegeben und in $8 \%$ wurden sie prophylaktisch verabreicht. Bei 33\% der Kinder wurden sie als OTC-Präparate gekauft und bei 26 bzw. 23\% vom Arzt oder Heilpraktiker verschrieben. Kinder, deren Mütter Hochschuldbildung hatten, erhielten signifikant häufiger Homöopathika.

Kommentar: In der Homöopathie wird gelehrt, dass Kinder besonders gut auf diese Therapieform ansprechen. Daher verwundert es kaum, dass Kinder häufig Homöopathika erhalten. Der Schönheitsfehler liegt in der fehlenden Evidenz: Wir haben vor Jahren gezeigt, dass in der Gesamtschau die klinischen Studien die o.g. Annahme nicht bestätigen. Besonders bedenklich stimmt mich der häufige Einsatz von Homöopathika in der Prophylaxe. Viele Homöopathen empfehlen „homöopathische Immunisation“ als Al-

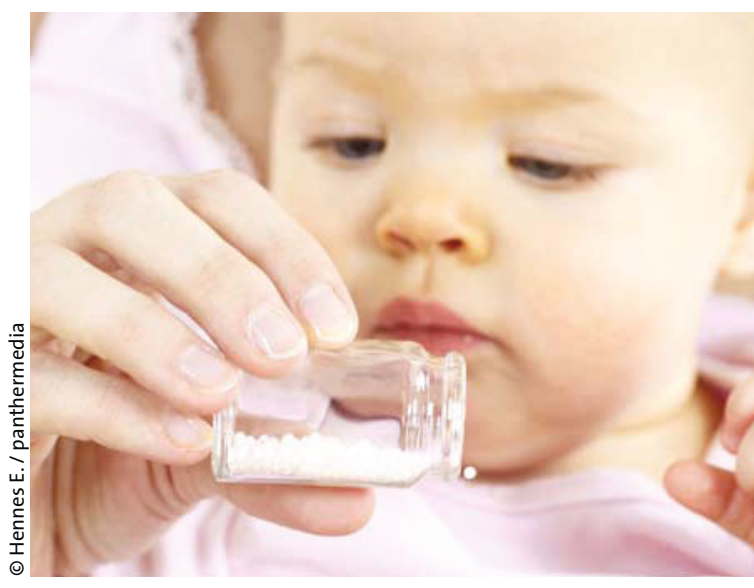

Globuli liegen bei Eltern im Trend.

ternative zum Impfen. Da auch hier kein Wirksamkeitsnachweis vorliegt, ist dieses Verhalten aus meiner Sicht unverantwortlich.

Prof. Dr. Edzard Ernst

Du Y, Knopf H. Paediatric homoeopathy in Germany: results of the German Health Interview and Examination Survey for Children and Adolescents (KiGGS). Pharmacoepidemiol Drug Saf 2009; 18: 370-9 